\title{
SALUD PUBLICA Y DISCAPACIDAD: Una mirada alternativa
}

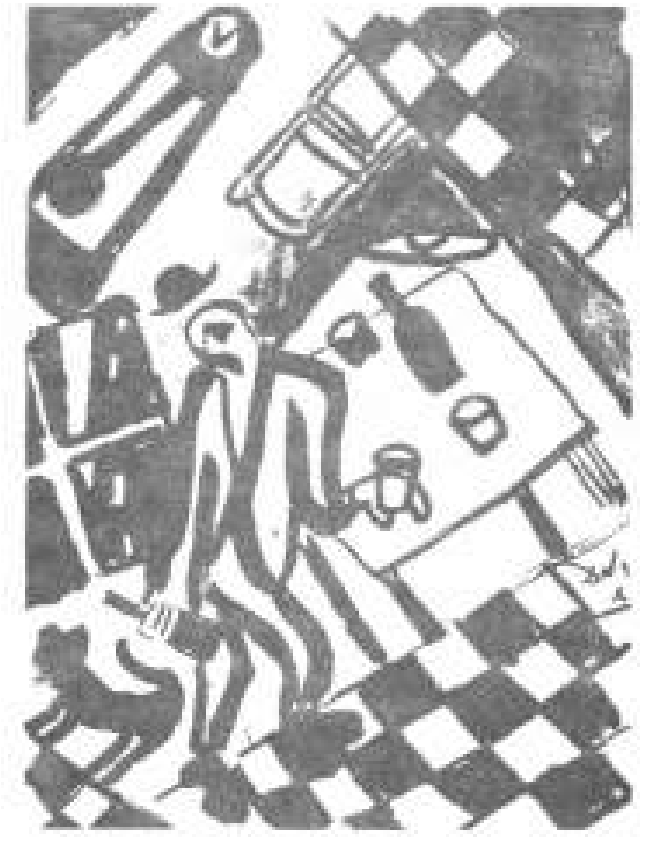

JOSE RUBÉN CASTILLO GARCIA

Trabajador Social. Mg, en Desarrollo Educativo y Social. Profesor Faculiad de Fisioterapia

Universidad Autónoma de Manizales CONSUELO VELLEZ ALVAREZ

Enfermera - Epidemióloga. Profesora Facultad de Fisioterapia y Odontología

Universidad Autónona de Manizales
T NTRODUCCIÓN:

Hablar de Salud Pública y Discapacidad Implica tener en cuenta las diferentes concepciones que se han desarrollado a través de la historia frente a ambas temáticas, para de esta manera, poder visualizar los retos a los cuales nos entrentaremos y en los que debemos comprometernos no sólo como profesionales de la salud, sino como actores vivenciales del proceso que tiene que ver con asumir la vida y sus posibilidades de una manera integral y no fraccionada, buscando siempre el logro de la salud, lo cual implica tomar posiciones frente a los mismos.

En principio, asumimos la salud como un fenomeno complejo que trasciende lo individual y to 
biológico, y la salud pública como todas aquellas actividades colectivas encaminadas a lograr mejores condiciones de vida en una población. La salud, inicialmente fue vista desde lo meramente clínico e implicaba reconocer las enfermedades, sus signos y sintomas e intervenir sobre un proceso patológico ya instaurado, posteriormente se consideró que lo importante era reconocer lo etiológico, entonces el estudio de las enfermedades se centró en buscar las causas de las mismas. pero más pensando en enfermedades transmisibles infectocontagiosas que en las no transmisibles: luego estos 2 elementos se complementan involucrando otro componente, el ecológico, en donde se piensa que cualquier enfermedad es multicausal, y que en ello juega un papel importante la triada ecológica (agente, huésped y ambiente). Esto sirvió de apoyo para que la Organización Mundial de la Salud definiese la salud como un "completo estado de bienestar físico, mental y social".

Esta mirada, sigue viendo la salud desde la enfermedad. es lo que tradicionalmente se ha manejado, por ello, los indicadores básicos han sido la morbilidad y la mortalidad, y por ende lo que finalmente reflejan son procesos mórbidos y no los saludables.

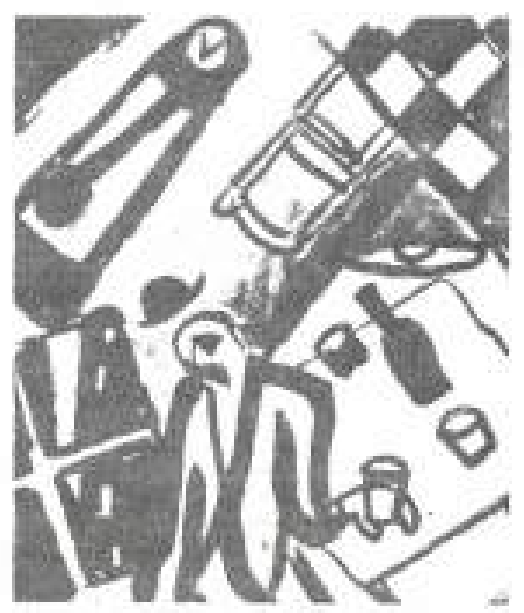

Lo dicho, permite plantear que el fenómeno salud-enfermedad se ve influenciado por los puntos de vista que se han tenido acerea del mismo, por los contextos políticos y económicos que se inscriben en la sociedad en la cual se ha movido.

\section{SALUD PUBLICA: OTRA MIRADA}

Retomando el texto aTeoria y Práctica de la Salud Públicar, elaborado por el Dr. Saúl Franco, vemos la salud pública desde una perspectiva diferente, el hecho nos invita a ver esie fenómeno desde diversas opciones: como ser y como manera de ser, como un saber y cómo un bacer. En razón de esto desarrollaremos a continuación una serie de conceptos que servinín para explicar el feno. meno de la salud publicu desde esta manera de comprenderio.

La salud pública planteada como ser, cómo situación y cómo manera de vivir, se asume como una manera de ser colectiva. Ello lleva a decir que hay salud publica donde exista un colectivo que se sienta bien y que se relacione con la naturaleza, que establezca un conjunto de relaciones entre sí, que le permita a cada uno $\mathrm{y}$ al conjunto sentirse alegre, sano; feliz, hasta donde sea posible, ser feliz dentro de una realidad histórica.

La salud pública debe encontrar un espacio de rea lización concreto, en el cual lo público deje de ser una abstracción y se convierta en la forma de rea. lización del ser social. La salud puiblica es una sensación, una percepción de que la relación colectiva funciona, de que es posible relacionamos, más no destruimos.

El objeto de la salud pública es justamente el conocimiento de las condiciones, de las posibilidades, de la dinámica de la realización del bienestar colectivo. La construcción de este objeto del conocimiento colectivo social implica rigurosidad cientifica, no podemos seguir tratando lo social con 
esa ligereza con la que tratamos las cosas cuando no las sabemos, con lo social hemos hecho una terrible trivialización y ello le ha quitado profundidad y aún más que profundidad. le ha quitado la posibilidad transformadora que tiene ese saber.

Cientificidad no es solo to cualitativo, hay cientificidad cuando existe la posibilidad de trascender la particularidad, de llegar a ese territorio que la apariencia nos esconde. Las relaciones de apariencia no deben generar relaciones de causalidad.

Otro aspecto a tener en cuenta es que la salud publica es un saber interdisciplinario. pot lo tanto. nos hace necesario romper con el saber parcial, médico en el cual se ha marcado siempre la salud pública, $y$ esto significa que la salud pública no sólo la hacen los salubristas, es responsabilidad y compromiso de todos.

El saber de la salud pública debe tener además una referencia epistemológica, no es el asunto de la verdad, del rigor, de la sistematicidad, es el asunto de los caminos de los procesos, del cómo lograr la comprensión en los fenómenos que la gente vive. bajo esta perspectiva nos debe preocupar el camino, la posibilidad de transformación.

La salud pública desde el saber nos debe permitir la interiorización, debe coadyuvar en la conciencia colectiva, facilitar el conocimiento de patrones, normas, maneras de asomarse a la vida, de relacionarse unos con otros, debe ser conciencia y camino de conciencia colectiva.

Desde el hacer, la salud pública es un hacer con múltiples campos de acción, los saberes acerca de la realidad, pueden finalmente ser convertidos en campos de acción, en posibilidades de cambio, en generar pautas, conductas, políticas, es decir, la salud pública es también un territorio para la acción.

Es entonces cuando se plantea que un salubrista integral tiene necesariamente que ser un vector. un medio $y$ un posibilitador de la transformación social y es asi como desde los muitiples haceres debe buscar la consolidación de condiciones saludables. posibilidades de vida y condiciones de convivencia, y en este aspecto vale la pena hacer una reflexión frente a lo que realmente es la convivencia, porque ésta por una parte es le forma como la vida se realiza, y al mismo tiempo como la vida se potencia en la interacción de cada persona.

Hay aquí un gran campo de acción entre la saiud pública y el Estado, y esto tiene que ver con aspectos como definición de políticas, asignación de recursos, priorización de inversiones, focalización de los gastos, explicación a los fenómenos colectivos entre otros muchos aspectos.

Bajo esta perspectiva, la principal tarea hoy de la salud pública es la defensa de fa vida, puesto que no se necesita que solamente se administren servicios, pues aunque existan muchos, estos hay que cualificarios generando con ellos condiciones y posibilidades dignas para la vida, y cuando hablamos de este aspecto es necesario pensar en la posibilidad de abordar los conceptos de cquidad, accesibilidad que, sin lugar a dudas, abren el camino para que más gente se beneficie y se involucre en el proceso, y en este sentido cobraria reaimente

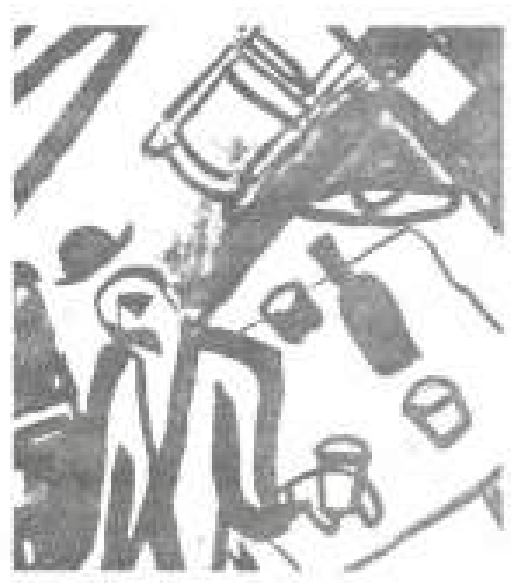


importancia, esto nos acercaría a generar en el colectivo condiciones de mayor bienestar.

Para generar estas condiciones la sałud pública se debe explorar otro aspecto que para este caso es la relaciôn hombre - naturaleza, es en esta perspectiva donde se garantiza en gran medida la conservación y la sobrevivencia de la especie, explorando cuál es el significado de mi transformación por el ambiente y del ambiente por mi.

La salud pública debe abordarse además como un hacer político, como espacio de construcción ciudadana, donde cada uno de los actores asuma su responsabilidad frente a la buisqueda de mejores condiciones que lleven al bienestar; $y$, qué es bienestar? Desde la salud pública cl bienestar es asumido como un saber que se comvierte en conciencia transformadora, que abre horizontes y que muestra a la sociedad la forma de evitar factores que lo afectan y de recortar inequidades.

A continuación desarrollaremos el concepto de discapacidad retomando los mismos aspectos anteriormente citados, que nos permitan entrelazar ambas temáticas para entender los retos a los cuales se hizo referencia al inicio de esta presentación.

\section{LA DISCAPACIDAD}

De manera similar que la salud y la salud pública han obedecido históricamente a las concepciones, los puntos de vista, los paradigmas, al nivel de conocimiento, a los intereses, a las cuituras y demás aspectos que permiten comprender los sentidos de los conceptos y las prácticas acerca de los mismos. la discapacidad también ba sido vista de diferentes manems a lo largo de la historia, teniendo como base la forma como se han visto las limitaciones físicas.

\section{Antecedentes}

En la Antiguedad Clásica, cuando se hicieron evidentes los aportes de las culturas griega y romana, la necesidad de formar ciudadanos para la guerra, hizo que se preocuparan por la conservación y el mantenimiento de los cuerpos en condiciones óptimas.

En la Edad Media, el aspecto religioso condicionó las formas de ver la salud y la enfermedad. Se privilegiaron los dogmas y por ello la ciencia dio paso a las consideraciones que explicaban las enfermedades a partir de castigos divinos. Los fimitados, fueron motivo de verguienza y de marginamiento social.

Tal como puede observarse, a lo largo de la historia, la salud se ha asumido como una situación que compete a la sociedad en su conjunto y la manera de explicar los fenómenos que tienen relación con ella, involucran prácticas y comportamientos que tiene aplicabilidad en el colectivo, es decir, la salud ha sido vista como un fenómeno público, lo cual nos permite decir que las limitaciones y los que las padecen han sido objeto de la forma de ver y de entender la sociedad la salud.

\section{«Desde la salud pública el bienestar es asumido como un sa-}

ber que se convierte en conciencia transformadora, que abre horizontes y que muestra a la sociedad la forma de evitar factores que lo afectan y de recortar inequidades.» 


\section{*... a lo largo de la historia, la salud se ha asumido como una situación que compete a la sociedad en su conjunto y la mane- ra de explicar los fenómenos que tienen relación con ella....}

En el Renacimiento se presentan acontecimientos que contribuyen a mirar este fenómeno de otra manera, se asume el ser humano corno el centro del mundo, esto permitió que se diese ruptura al oscurantismo y se posibilitara la investigación tendiente a descubrir la razón de ser de los fenómenos de la vida en general y de la salud en particular, en principio, apoyados en hechos tangibies y posteriormente en la razón. Además de to anterior, los inventos $\mathrm{y}$ avances tecnológicos favorecieron la divulgación de los conocimientos obtenidos por estas vias, y que se asumiera como responsabilidad de la sociedad, en su conjunto. lo que sucediese con las personas enfermas, limitadas y con alteraciones fisicas. Entra a tener impulso y preeminencia el empirismo y por ende, a valorarse los aportes cientificos anatómicos, aisiando. segregando y seccionando lo físico de los demás componentes del ser humano, distanciando $y$ hasta olvidando los aspectos sociales y culturales que habian sido privilegiados en los puntos de vista anteriores.

Coincide esta situación con el surgimiento del Capitalismo, con la racionalidad de la productividad, de la ganancia, con la división especializada de los oficios, con la idea de la utilidad y de la ganancia como formas de hacer rentables los roles sociales.

Con este marco de referencia, acontece la presencia progresiva de corrientes estructuralistas (siglo XV y XVI), fisiologicas (siglo XVII y XVIII) que se enmarcan dentro de la perspectiva clínica de la salud y tal como se indicó antes, de la caracterización de los fenómenos de la salud con base en los signos y en los sintomas de las enfermedades. Además de estos aspectos, la creciente importancia de la actividad laboral industrial y los fenomenos patológicos relacionados con situaciones de trabajo, así como los descubrimientos de Luis Pasteur. llevan la mirada hacia la etiologia de las enfermedades, luego a la comprensión de la importancia de los factores sicológicos y sociales y a la progresiva conciencia acerca de la pluralidad de factores relacionados con el fenómeno de la salud, la integralidad como también a lo que estamos viviendo en la actualidad con la propuesta de asumir dicho fenómeno como algo complejo en su ser. interdisciplinario en su saber e interprofesional en su bacer.

\section{La Discapacidad en la actualidad}

En los albores del siglo XXI, el fenómeno de la discapacidad se ve influenciado básicamente por dos perspectivas que han coexistido a lo largo de la historia de las concepciones sobre la salud y la enfermedad. De un lado, aquelia que ha servido de fundamento y que se evidencia en la Clasificación Intemacional de Deficiencias, Discapacidades y Minusvalias - CIDDM-, aceptada y avalada por la Organización Mundial de la Salud-OMS-, (desde 1980) y tal como ellos mismos expresan, se orientó a plasmar un manual de clasificación de las consecuencias de la enfermedad. De otro, la propuesta denominada Clasificación Internacional del Funcionamiento y la Discapacidad -CIDDM2. "orientada a clasificar sistemáticamente los es. tados funcionales asociados a los estadus de salud, en la perspectiva de brindar un lenguaje unificado y estandarizado que sirva para describir el 
funcionamiento humano $y$ la discapacidad como elementos importantes de la sajud ${ }^{+1}$

En el primer caso, es amplia la experiencia y la tradición en cuanto a la forma de caracterizar la discapacidad en las poblaciones humanas a nivel local, regional y nacional, en tanto que la segunda está en vía de experimentación de validación y de discusión hasta el 2001, año en el cual espera sis: tematizar los resultados y proponerla como modelo oficial de la - O.M.S.- para sus filiales a nivel mundial.

La CIDDM-Clasificacion Internacional de Deficiencia, Discapacidad y Minusvalia.

Tal como se ha indicado antes, desde esta perspectiva, se hace énfasis en la enfermedad como criterio de medición de la situación de los sujetos. y tiene como apoyo el estado que presenta el organismo a nivel de su constitución y de su funcionamiento físico, visto desde su estructura, su funcionamiento o de su relación con los demás.

Desde este punto de vista:

"La deficiencia representa la desviación de alga. na norma en el estado biomédico del individuo:... se caracteriza por pérdidas o anormalidades que pueden ser temporales o permanentes, e inclarye la existencia a aparición de una anomalia, defecto-péndida en una entremidad, of gano, tejido u otra estrictura carporal o un defecto en un sistema fiencional o mecanismo del cuerpo, incluyendo los xistemas de la funcion mental. "t

Como puede observarse, en esta definición se hace énfasis sobre los elementos estructurales y funcionales de los sujetos particulares, por ende lo co- lectivo, no va más alít de buscar los datos de generalización de lo que acontece con congiomera dos, es la sumatoria de casos que en un principio se captan aislados.

En reiación con la discapsicidad, lo que este punto de vista plantea que se refiere a:

".cualquier restricción o ausencia (debida a una deficiencia) de la capacidad para realizar una actividad en la forma o dentro del margen que se considera normal para un ser humano.... se refiere a actividades complejas o integradas que se esperan de la persona o del cuerpo en conjunto, como pueden ser las representadas por tareas. aptitudes y conductas, representir una desviación de la norma desde el punto de vista de la actuación como individuo, a diferencia de la del órgano o mecanismo. Lo caracteristico de este concepto son los excesos o defectos en relación con la conducta 0 actividad que, normalmente, se espera y que pueden ser temporales o permanentes, reversibles o irreversibles, progresivos o regresivos. La cancterística fundamental es la objetivación. Este es el proceso por el cual una limitación funcional se manifiesta como una realidad en la vida diaria, con lo cual el probiema se hace objetivo al interferir las actividades corporales".?

Aunque este concepto, está referido en cuanto objetivación a la acción del sujeto a lo físico y lo social, en cuanto conductas que transgreden lo es- 
perado dentro de un criterio de normalidad del individuo. La perspectiva está dada en función de la práctica de éste, más que de lo que le acontece como situación que puede afectar lo público.

Con respecto de la minusvalía, se indica que corresponde $\mathrm{a}$ :

*.. una situación desventajosa para un individuo determinado, consecuencia de una deficiencia o de una discapacidad, que limita o impide el desempeño de un rol que es normal en su caso (en función de su edad, sexo, y factores sociales y culturales)

En este concepto hay tres características importantes que deben tenerse presente:

1) Se concede un valor a la desviación đe una forma estructural, funcional o de actuación, bien por parte del propio individuo o de sus semejantes en el grupo con que se relaciona.

II) La valoración depende de las normas cuiturales, de tal manera que una persona puede ser minusválida en un grupo y en otro no -el tiempo, lugar, status, y rol son todos elementos con los que hay que contar.

III) De primera instancia, la valoración suele ser en desventaja del individuo afectado.

La condición de minusvalido hace referencia a otras personas, de ahí la importancia de los valores sociales existentes, los cua- les. a su vez están influenciados por la organización instimcional de la sociedad.

.. La minusvalía se caracteriza por la discordia entre la actuación y el estatus del individuo y las expecutivas del grupo concreto al que pertenece ${ }^{\text {ru }}$

Según lo planteado, la presencia de la discapacidad se manifiesta con base en los registros, brindando predominio de los datos cuantitativos en la presentación de la información, es por ello, que se nos generan algunas inquietudes desde el punto de vista de nuevas posibilidades del abordaje de la salud públici, algunas de las cuales pueden ser atendidas en la caracterización que propone la nueva clasificación de la discapacidad propuesta por la O.M.S. de tal manera que este fenómeno sea observado mas desde la salud puiblica integral y compleja que desde la perspectiva tradicional, de los individuos como usualmente se ha trabajado. Veamos algunos criterios que hacen referencia a los alcances de la nueva propuesta.

La CIDDM-2-Clasificación internacional del Funcionamiento y la Discapacidad.

Esta clasificación se orienta â establecer criterios y pautas para caracterizar el Funcionamiento y la Discapacidad de las personas (no de las deficiencias, discapacidades y minusvalias), concentrando esfuerzos en establecer las capacidades motoras de los sujetos. Un asunto interesante de tener en cuenta en esta nueva clasificación, es que el término minusvalía ha sido excluido y el de discapacidad se utiliza para dar cuenta de tas tres dimensiones que se contemplaban antes. Es de 
anotar que este término ha sido modificado por el concepto de "limitación en la actividad", al parecer con la intención de hacer énfasis en los sentidos que expresan el comportamiento humano.

Algunos de los conceptos básicos utilizados en esta clasificacion, son asumidos como:

"Funciones corporales: Son las funciones fisiológicas y psicológicas de los sistemas corporales. Las deficiencias son problemas en la función o en la estructura del cuerpo, tales como la desviación o una pérdida significativa.

Estructura Corporal: Estructuras corporales y partes anatómicas del cuerpo tales como los órganos, las extremidades y sus componentes. Las deficiencias son problemas en las funciones o estructuras corporales, como por ejemplo una desviación o perdida significativa de las mismas

Actividades: Es la realización de una tarea o acción por un individuo, las limitaciones en la actividad son las dificultades que un individuo puede experimentar al realizar actividades.

Participación: Es el acto de involucrarse de un individuo en situaciones vitales, en relación con estados de salud, funciones y estructuras corporales, actividades y fac- tores contextuales. Las restricciones en la participación son problemas que un individuo puede experimentar en la manera o el grado de involucrarse en situaciones vitales. (Este elemento es posiblemente to que queda de la anterior ciasificación con respecto de la minusvalia?")

Factores contextuales: Forman el ambiente físico, social y actitudinal en el cual la gente vive y dirige sus vidas. ${ }^{\text {th }}$

Son varios los criterios que permiten diferenciar la CIDDM y la CIDDM-2-, entre los cuales se pueden destacar:"

1. En la primera se busca registrar la interacción del individuo y su entorno para la apreciación de sus capacidades, en tanto que en la seganda se busca agrupar las consecuencias asociadas con las condiciones de salud determinadas por las dimensiones de estructura of unción corporal, actividades personales y participación en sociedad.

2. El objetivo principal de la versión de 1980 , es el de determinar el grado de funcionalidad de las personas con relación al entorno. La propuesta, de la segunda se refiere a buscar la construcción de un lenguaje común y práctico para cualquiera que tenga injerencia en el tema de la discapacidad y plantear términos funcionales, consecuencia de las condiciones de salud asociadas a nivel corporal, individual y social.
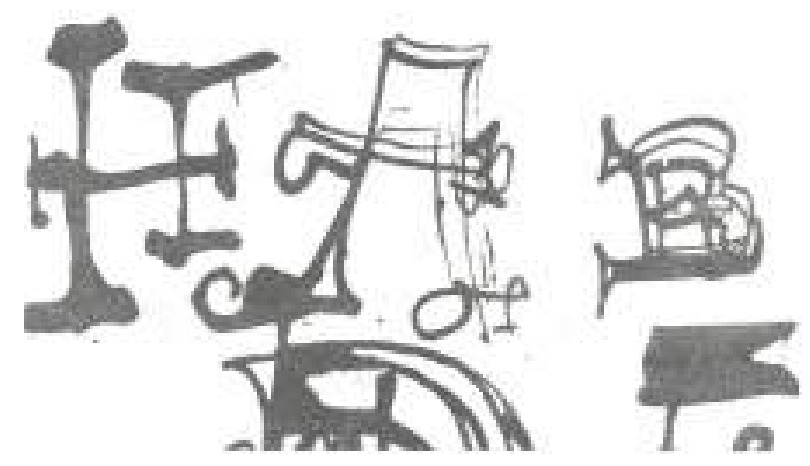


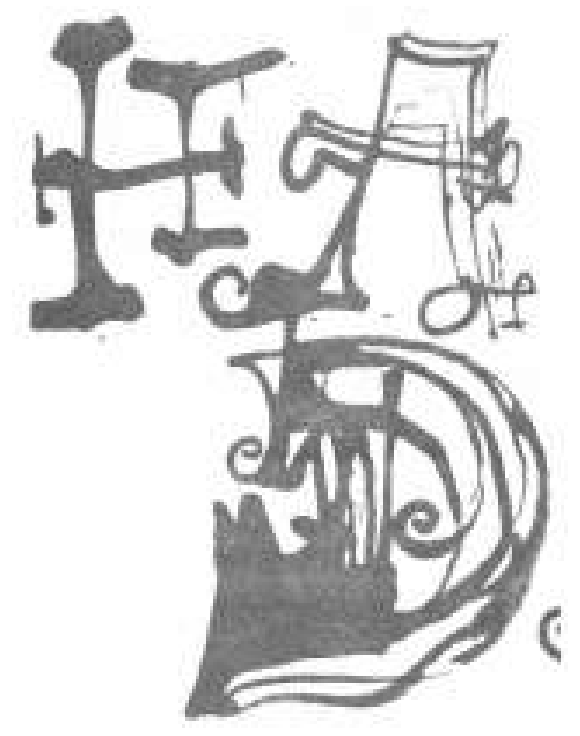

3. La primera versión de la clasificación, busca reflejar la consecuencia de la deficiencia en cuanto a capacidad funcional y actividad de la persona. La otra, la propuesta, considera necesario cambiarle el nombre a la discapacidad llamándola limitación de la actividad, ya que tiene en cuenta la naturaleza y calidad para la ejecución de actividades, además aporta, en el sentido de plantear que el uso de dispositivos de ayuda no elimina la deficiencia pero si puede eliminar las limitaciones de la actividad.

4. La primera clasificación considera la minusvalía como la alteración para el desempeño de un rol social, laboral, familiar de un individuo como resultado de la discapacidad o deficiencia. La propuesta moderna, cambia el nombre de minusvalía por el de restricción en la participación, la cual puede estar restringida en naturaleza, durnción y calidad, además. incorpora como un estándar internacional "la igualdad de oportunidades".

\section{A MANERA DE CONCLUSIÓN}

Por lo visto, las nuevas perspectivas en cuanto a la manera de interpretar y de valorar la discapacidad, invitan a ampliar la visión que se tenía en el pasado frente a este fenomeno. Se percibe el deseo de trascender lo orgánico en cuanto a to estructural y lo funcional, como fundamento de la caracterización de la discapacidad, para verlo como un asunto de restricción y de limitación en la actividad en sí misma para el sujeto, pero en la perspectiva de su relación con los demás, lo cual involucra la vinculación a las actividades vitales en la sociedad, en calidad de ser participe de clia.

Además, se vislumbra la necesidad de que el análisis de esta temática, asuma referencias diferentes, se distancie de la fragmentación de aspectos de la realidad humana tales como los estructurales y los funcionales, y se comprende el funcionamiento humano como un fenómeno que integra diversos componentes y en el cual su realidad es compleja, por sí misma.

La referencia cambia de lo físico en abstracto, aislado de los demás componentes del ser humano. se trata de referirlo al ser humano real, complejo. diverso, muitifactorial e interrelacional contextuado en su propia vida, en una situación histórica especifica, es decir, vivenciando un mundo que le ubica en el tiempo y en el espacio, y a a partir de ello que puede ser entendido en su intereses, intencionalidades y sentidos de vida. Este es el ser humano, susceptible de tener limitaciones en su comportamiento y en su expresión consigo mismo y con los demás.

Con base en lo anterior, se puede plantear que para hablar del fenómeno que usualmente se ha denominado discapacidad, debernos remitimos al lenguaje corporal que tenga la persona, tratando de referirse no solamente a la funcionalidad en la realización de las actividades sino a la capacidad del cuerpo para expresar sus necesidades y sentidos con los cuales se desempeña. 
En el mismo sentido, la comprensión del movimiento corporal humano como elemento importante para la vida del hombre y como la consecuencia de su alteración lleva a la limitación en la actividad, refiriéndose no solo al significado del término, sino al sentido $y$ a las concepciones que este abarca.

Lo dicho. lleva a pensar que son muchos los aspectos y situaciones que se deben tener en cuenta para efectos de caracterizar la situación de la discapacidad en la perspectiva de la salud pública. En razón de ello se ve necesario centrarnos en la situación de vida de las poblaciones, en las formas de saber, de sentir y de hacer de las personas, además de las características de las relaciones que establecen con los demás, es decir, en las circunstancias en que construyen sociedad.

Esto, involucra el manejo de diversas categorías. tal como se propone en la CIDDM-2-, que empieza por la identificación de lo que acontece con las estructuras corporales, las funciones corporales, las actividades, la participación, las circunstancias en que se desenvuelve el contexto.

Además, si se trata de validar lo propuesto en esta clasificación, vale la pena llamar la atención acerca de ciertos aspectos que deben ser tenidos en cuenta, para evidenciar el comportamiento de la "discapacidad", de la "limitación en la actividad" o "limitación del movimiento", es por ello, que se proponen entre otros. Ios siguientes interrogantes. para que sean abordados en el proceso de evaluación de este fenómeno:

\section{RETOS DE LA UNIVERSIDAD FREN- TE AL FENOMENO DE LA SALUD PÚBLICA Y LA DISCAPACIDAD}

El Dr. Saúl Franco en el documento al cual nos hemos referido plantea una seric de interrogantes frente a las posibles respuestas de las facultades, escuelas, y departamentos de salud. que consideramos necesarias retomarlas al final de este documento.

Contribuir a crear un nuevo saber.

- Ir a los escenarios en los cuales acontece la vida de la gente,

- Encontrar allí nuevas maneras de saber.

- Salir de la cientificidad cuantitativa.

- Ampliar la visibilidad probiemática.

- Romper con lo inmediato, buscar la explicación (y la comprensión), a partir de ello, convocar a la acción. de esta manera se genera una salud pública viva.

- Innovar los mecanismos de transmisión del saber.

- Dar respuesta más a las prioridades sociales, qủe a la exigencia intraacadémica. $\propto$ No es tanto el tema que la disciplina pide, sino cual es el tema que la sociedad palpita:.

- Crear contenidos de salud publica no solo para el Médico o para el salubrista, sino para cualquier ciudadano, para cualquier nivel

- Los salubristas nos relacionamos con ciudadanos y sí seguimos con la añoranza del paciente, vamos a perder de vista las dimensiones enormes que se abren cuando no hay relación médico paciente.

- La salud pública tiene que perder el miedo a otros saberes, a otras maneras de entender la salud, a otras prácticas sociales.

- Se trata de tener éticas de vida: Pedagógica, ciudadana, religiosa, metafísica, social, convivencia; respeto por la diferencia. 
- Ayudar a recomponer lo que podemos llamar un nuevo pacto social en y por la salud, que permita:

- Interpretar que es lo que le interesa a la colectividad.

- Que es los que necesita para estar bien.

- Reinterpretar las relaciones de poder del establecimiento sanitario.

- Nuevas reglas de juego.

- Nueva epistemología.

Todo esto contribuiría a darle a la salud pública la oportunidad necesaria para el logro de su gran objetivo, las condiciones $y$ calidad de vida del colectivo.

\section{BIBLIOGRAFIA}

AGUDE1,O, Martinez, Ayda Zulamy y otras "Caracterización de la situación fatworal

y de las tarreras arquiteciónicas y urbanisticas que limitan ef desplaramiento al sitoo de trabajo, seguin el grado de dificultad que ofrecen estas a las personas cin discapracidad de locumocion, que pertesecen a frateraidad y la asociación de personas con discapacidad. junio de 1986. En ta Ciodad de Manizales

GOMEZ, Asgela Vivana y otros "Reser̂a de la Conseruccicin Teorica en torno a la Discapacidad", Universidad Auacnoms de Manezales, Faculad de Fisicierapia. Febrero de 2000 .

ORGIANLZACION MUNDIAL DE LA SALUD-O.M.S - Clasificación Internacional del Funcionamiento y la Discapacidad. CIDDM-2- Borrsder, Beta 2 Versićn completa Gitnebra, 1999

ALCALDIA DE MANEZALES, SECRETARIADESALUD, Ca sificacion Intemacional de Deficiencias, Discapacidaden y Minusvalias", Manizales 1991. Exte documento es traducción dei de la O.M.S.
ORGANIZACTON MUNDLAL DE LA SALIPDG,M.S-Clasif. cacicin Internacional del Frencionaumiento y ta Discapacidad. CIDDM-2. Borrador, Beta 2 Versićn completa Ginebra, 1999.

FRANCO, Sail. Tecria y Practuca de Sal ud Puiblica. Uiniversadad de Antiocuia Facultad Nacional de Salud Publica. Modeltin 1993.

'ORGANIZACION MUNDIAL DE LA SALUD-

O.M S - Clasificación internacional del Funcionamiento y la Discapacidad. -CIDDM-2-Borrador.

Bata 2. Versión completa Ginebra, 1999

2 ALCALDIA DE MANIZALES, SECRETARIA DE SALUD Clasificación Internacional de Deficiencias. Discapacidades y Minusvalias", Manizales 1991. Este documento es traducción del de la O.M.S pág 57

${ }^{7}$ Ibid. Pag. 59

4 ibid, pag. 59 y 60

"Este comentano es nuestro

- ORgANizACIÓN MUNDIAL de LA SALUdO.M.S. - Clasificación Internacional del Funcionamiento y la Discapacidad-CIDDM-2-Borrador, Beta 2. Versión completa Ginebra, 1999, paginas $44,84,101,145$ y 169.

' GOMEZ, Angela Viviana y otros "Resenta de la Construcción Teorica en torno a la Discapacidad" Universidad Autónoma de Manizales, Facultad de Fisioterapia. Febrero de 2000 